Pacific Journal of Mathematic 


\title{
ON A CORRECTNESS CLASS OF THE BESSEL TYPE DIFFERENTIAL OPERATOR $S_{\mu}$
}

\author{
W. Y. LEE
}

We have found a correctness class of the Bessel type differential operator $S_{\mu}=\partial^{2} / \partial x^{2}-\left(4 \mu^{2}-1\right) / 4 x^{2}$ among smooth functions using the Hankel transform and linear mapping theory. It is left as an open problem to find its correctness class among nonsmooth functions satisfying certain boundary conditions.

1. Preliminary. This is a continuation of our previous paper [10]. In [10] we investigated a uniqueness class of the Cauchy problem of the differential operator $S_{\mu}=\partial^{2} / \partial x^{2}-\left(4 \mu^{2}-1\right) / 4 x^{2}$, and left out a question on the genus of $S_{\mu}$. In this paper we answer the question (Lemma 2.2); in fact the genus turned out to be less than that of the differential operator $i \partial / \partial x$, disproving our previous conjecture ([10]). We also find a correctness class of the same operator $S_{\mu}$, using the same notations as in [9] or [10]. Let us start with the definitions of the spaces $B_{\mu, b}, H_{\mu, \alpha}^{\beta}$ and $Y_{\mu, b}^{2 r}$.

Definition 1.1. For any real numbers $\mu$ and $b>0$, the space $B_{\mu, b}$ consists of smooth functions $\varphi$ such that $\varphi(x)=0$ for $x>b$ and satisfies the inequalities

$$
\gamma_{b, k}^{\mu}(\varphi)=\sup _{0<x<\infty}\left|\left(x^{-1} D\right)^{k}\left(x^{-(\mu+1 / 2)} \varphi(x)\right)\right|<\infty, \quad k=0,1,2, \cdots
$$

Definition 1.2. For any real numbers $\mu, \alpha>0$ and $\beta>0$, the space $H_{\mu, \alpha}^{\beta}$ consists of smooth functions on $0<x<\infty$ for which the inequalities

$$
\begin{array}{r}
\delta_{k, q}^{\mu}(\varphi)=\sup _{0<x<\infty}\left|x^{k}\left(x^{-1} D\right)^{q}\left(x^{-(\mu+1 / 2)} \varphi(x)\right)\right| \leqq C A^{k} B^{q} k^{k \alpha} q^{q \beta} \\
k, q=0,1,2, \cdots
\end{array}
$$

are satisfied where $k^{k \alpha}=1, q^{q \beta}=1$ for $k, q=0$ and the constants $C, A$ and $B$ depend on the testing function $\varphi$.

Definition 1.3. For any real numbers $\mu, r>0$ and $b>0, \Phi \in Y_{\mu, b}^{2 r}$ if $z^{-(u+1 / 2)} \Phi(z)$ is an even entire function for which the inequalities 


$$
\nu_{b, k}^{\mu, 2 r}(\Phi)=\sup _{z=x+l y} \mid e^{-b|y|^{2 r}}\left(z^{2 k-\mu-1 / 2} \Phi(z) \mid<\infty \quad k=0,1,2, \cdots\right.
$$

are satisfied. Here $z^{-(\mu+1 / 2)} \Phi(z)$ is understood to be a principal value.

The topology of the spaces $B_{\mu, b}, H_{\mu, \alpha}^{\beta}$ and $Y_{\mu, b}^{2 r}$ are generated by the seminorms $\left\{\gamma_{b, k}^{\mu}\right\}_{k=0}^{\infty},\left\{\delta_{k, q}^{\mu}\right\}_{k, q=0}^{\infty}$ and $\left\{\nu_{b, k}^{\mu, 2 r}\right\}_{k=0}^{\infty}$ respectively. It is easy to see that all three spaces are Fréchet spaces.

From the definitions, we immediately have:

LEMMA 1.1. If $b_{1}<b_{2}, \alpha_{1}<\alpha_{2}$ and $\beta_{1}<\beta_{2}$, we have $B_{\mu, b_{1}} \subset B_{\mu, b_{2}}$, $H_{\mu, \alpha_{1}}^{\beta 1} \subset H_{\mu, \alpha_{2}}^{\beta_{2}}$ and $Y_{\mu, b_{1}}^{2 r} \subset Y_{\mu, b_{2}}^{2 r}$.

Suppose $\varphi$ belongs to the Zemanian space $H_{\mu}$. Utilizing [9; p. 337], the space $H_{\mu, \alpha}^{\beta}$ is characterized by the following inequalities.

LEMma 1.2. $\varphi \in H_{\mu, \alpha}^{\beta}$ if and only if $\varphi$ satisfies the following inequalities.

$$
\left|D^{q}\left(x^{-\mu-1 / 2} \varphi(x)\right)\right| \leqq C_{q} B^{q} q^{q \beta} \exp \left(-a^{\prime} x^{1 / 2}\right), \quad q=0,1,2, \cdots
$$

where $a^{\prime}$ is a positive constant less than $a=\alpha e^{-1} A^{1 / \alpha}$.

Let $\boldsymbol{h}_{\mu}$ for $\mu \geqq-1 / 2$ be the conventional Hankel transform defined by $([17 ;$ p. 561])

$$
\left[\boldsymbol{h}_{\mu} \varphi(x)\right](y)=\int_{0}^{\infty} \varphi(x) \sqrt{x y} J_{\mu}(x y) d x
$$

where $J_{\mu}(x)$ is the Bessel function of the first kind. Then we restate the following theorem from [10]:

THEOREM 1.1. For any real numbers $\mu \geqq-1 / 2$ and $r>0$, the Hankel transform $\boldsymbol{h}_{\mu}$ is an isomorphism from the space $B_{\mu, b}$ onto the space $Y_{\mu, b}^{2 r}$.

2. Parabolic systems. I. M. Gelfand and G. E. Shidov ([3; pp. 105-164]) investigated a correctness class of parabolic equations of the differential operator $i \partial / \partial x$ such that

$$
\begin{gathered}
\frac{\partial u(x, t)}{\partial t}=P\left(i \frac{\partial}{\partial x}\right) u(x, t) \\
u_{0}(x)=u(x, 0)
\end{gathered}
$$


where $u(x, t)$ is an $m \times 1$ column vector, $P(\xi)$ is an $m \times m$ matrix in $\xi$ with constant coefficients, all the eigenvalues of $P(\xi)$ are even powers of $\xi, x=\left(x_{1}, \cdots, x_{m}\right) \in R^{m}$ and $t \in R$. The Fourier transform of the system (2.1)-(2.2) yields

$$
\begin{aligned}
\frac{\partial \hat{u}(\xi, t)}{\partial t} & =P(\xi) \hat{u}(\xi, t) \\
\hat{u}_{0}(\xi) & =\hat{u}(\xi, 0)
\end{aligned}
$$

where $\xi=\sigma+i \eta$. The formal solution of the system $(2.1)^{\prime}-(2.2)^{\prime}$ is given by

$$
\hat{u}(\xi, t)=\exp \left(t P(\xi) \hat{u}_{0}(\xi)\right.
$$

Let $p_{0}$ and $h$ be the reduced order and the parabolicity index respectively of the system $(2.1)^{\prime}-(2.2)^{\prime}$. Then according to [3; p. 41 and p. 114], the following inequalities hold:

$$
\begin{aligned}
\|\exp (t P(\xi))\| & \leqq C_{1} \exp \left(a t|\xi|^{p_{0}}\right) \\
\|\exp (t P(\sigma))\| & \leqq C_{2} \exp \left(-b t|\sigma|^{h}\right)
\end{aligned}
$$

where $C_{1}, C_{2}, a$ and $b$ are positive constants. Let $\rho$ be the genus of the given system. Then in a domain defined by

$$
|\eta| \leqq B_{1}(1+|\sigma|)^{\rho}, \quad \rho \geqq 1-\left(p_{0}-h\right)
$$

the inequality

$$
\|\exp (t P(\xi))\| \leqq C_{3} \exp \left(-b^{\prime} t|\sigma|^{h}\right)
$$

is satisfied where $b^{\prime}$ is arbitrarily close to $b$. Utilizing [2; p. 217], the inequalities (2.3) and (2.6) lead us to

$$
\begin{aligned}
\|\exp (t P(\xi))\| & \leqq C_{4} \exp \left(-b t|\sigma|^{h}+a^{\prime} t|\eta|^{p_{0} / \rho}\right) \\
& \leqq C_{4}^{\prime} \exp \left(a^{\prime} t|\eta|^{p_{d} / \rho}\right)
\end{aligned}
$$

where $a^{\prime}$ is a constant $\leqq B_{1}(a+b)$.

Let $S_{\mu}$ for $\mu \geqq-1 / 2$ be the Bessel type differential operator defined by $S_{\mu}=\partial^{2} / \partial x^{2}-\left(4 \mu^{2}-1\right) / 4 x^{2}$, and consider the Cauchy problem of the system (2.1)-(2.2) with $i \partial / \partial x$ replaced by $S_{\mu}$ :

$$
\frac{\partial u(x, t)}{\partial t}=P\left(S_{\mu}\right) u(x, t)
$$




$$
u_{0}(x)=u(x, 0)
$$

Since $P$ is an even polynomial, the system (2.1)-(2.2) is parabolic if and only if the system (2.8)-(2.9) is parabolic.

The Hankel transform of the system (2.8)-(2.9) gives ([20; p. 139])

$$
\begin{aligned}
\frac{\partial U(\xi, t)}{\partial t} & =P\left(-\xi^{2}\right) U(\xi, t) \\
U_{0}(\xi) & =U(\xi, 0)
\end{aligned}
$$

where $U(\xi, t)=\left(\boldsymbol{h}_{\mu} u(x, t)\right)(\xi)$. Obviously the formal solution of $(2.8)^{\prime}-(2.9)^{\prime}$ is given by

$$
U(\xi, t)=\exp \left(t P\left(-\xi^{2}\right)\right) U_{0}(\xi)
$$

An inspection of (2.3), (2.4), (2.5) and (2.10) show us

LEMMA 2.1. The reduced order and the parabolicity index of the system $(2.8)-(2.9)$ are $2 p_{0}$ and $2 h$ respectively.

Let $\rho^{\prime}$ be the genus of the system $(2.8)^{\prime}-(2.9)^{\prime}$. Then from the inequalities (2.5) and (2.6), we get

Lemma 2.2. $1-2\left(p_{0}-h\right) \leqq \rho^{\prime}<\rho \leqq 1$.

Since $p_{0} \geqq h([3 ; \mathrm{p} .116])$, it follows that $\rho^{\prime}>0$ if and only if $p_{0}-h<1 / 2$. Now we shall prove the main theorem.

THEOREM 2.1. For any real numbers $\mu \geqq-1 / 2$ and $d>0$, let $u_{0}(x)$ belong to the space $B_{\mu, d}$. If $P$ is an even polynomial, and if the genus $\rho^{\prime}$ of the system $(2.8)^{\prime}-(2.9)^{\prime}$ is positive, then the correctness class of the system (2.8)-(2.9) belongs to the space $B_{\mu, d+a_{0}}$ for any $0 \leqq t \leqq T$, where $a_{0}=a^{\prime} T$, and $a^{\prime}$ is a positive constant to be determined.

Proof. Since the formal solution of the Hankel transformed system $(2.8)^{\prime}-(2.9)^{\prime}$ of $(2.8)-(2.9)$ is given according to $(2.10)$ by

$$
U(\xi, t)=\exp \left(t P\left(-\xi^{2}\right)\right) U_{0}(\xi)
$$

let us first estimate the norm of $\exp \left(t P\left(-\xi^{2}\right)\right)$.

Since the reduced order and the parabolicity index of the system $(2.8)^{\prime}-(2.9)^{\prime}$ is $2 p_{0}$ and $2 h$ respectively from Lemma 2.1 , we get from the inequalities (2.3) and (2.4) that 


$$
\begin{aligned}
\left\|\exp \left(t P\left(-\xi^{2}\right)\right)\right\| & \leqq C \exp \left(a t|\xi|^{2 p_{0}}\right) \\
\left\|\exp \left(t P\left(-\sigma^{2}\right)\right)\right\| & \leqq C_{1} \exp \left(-b t|\sigma|^{2 h}\right)
\end{aligned}
$$

Since the genus $\rho^{\prime}$ of the system $(2.8)^{\prime}-(2.9)^{\prime}$ is positive, an application of $[3 ;$ p. 115$]$ and the inequality $(2.6)$ reveal that

$$
\left\|\exp \left(t P\left(-\xi^{2}\right)\right)\right\| \leqq C_{2} \exp \left(-b^{\prime} t|\sigma|^{2 h}\right)
$$

where $b^{\prime}$ differs from $b$ by an arbitrarily small number. Utilizing [2; $\mathrm{p}$. 217], the inequalities (2.11), (2.12) and (2.13) lead us to

$$
\begin{aligned}
\left\|\exp \left(t P\left(-\xi^{2}\right)\right)\right\| & \leqq C_{3} \exp \left(-b^{\prime} t|\sigma|^{2 h}+a^{\prime} t|\eta|^{2 p_{0} / \rho^{\prime}}\right) \\
& \leqq C_{3}^{\prime} \exp \left(a^{\prime} t|\eta|^{2 p_{\alpha} \rho^{\prime}}\right) \\
& \leqq C_{3}^{\prime} \exp \left(a^{\prime} T|\eta|^{2 p_{d} / \rho^{\prime}}\right)
\end{aligned}
$$

where $a^{\prime}>a$ depends on $b, C$ and $C_{2}$. Let $a_{0}=a^{\prime} T$ and let $r>$ $p_{0} / \rho^{\prime}$. Then the inequality (2.14) shows that $\exp \left(t P\left(-\xi^{2}\right)\right)$ belongs to the space $\xi^{-(\mu+1 / 2)} Y_{\mu, a_{0}}^{2 r}$. Since every testing function in $Y_{\mu, d}^{2 r}$ is of a form $\xi^{\mu+1 / 2} \Phi(\xi)$, where $\Phi$ is an even entire function from Definition 1.3, and since $U_{0}(\xi)=\boldsymbol{h}_{\mu} u_{0}(x)$ belongs to the space $Y_{\mu, d}^{2 r}$ if $u_{0}(x) \in B_{\mu, d}$ from Theorem 1.1, it follows that $U(\xi, t)=\exp \left(t P\left(-\xi^{2}\right) U_{0}(\xi)\right)$ belongs to the space $Y_{\mu, d+a_{0}}^{2 r}$ from Lemma 1.1. Consequently, $u(x, t)=\boldsymbol{h}_{\mu}^{-1} U(\xi, t)$ belongs to the space $B_{\mu, d+a_{0}}$ by virtue of Theorem 1.1. Continuous dependence of the solution on the initial function $u_{0}(x)$ follows from the fact of uniform convergence of the inverse Hankel transform $\boldsymbol{h}_{\mu}^{-1} U(\xi, t)$ with respect to $t$. This proves the theorem.

REMARK. Since $T$ is any positive real number, the correctness class of the system (2.8)-(2.9) approaches to the Zemanian space $H_{\mu}$ as $T \rightarrow \infty$, because the space $B_{\mu, d}$ approaches to the Zemanian space $H_{\mu}$ as an inductive limit space ([19] and [20]) as $d \rightarrow \infty$.

Suppose the system $(2.8)-(2.9)$ is petrowsky-parabolic such that real parts of the eigenvalues of $P$ are bounded by a negative constant for $|\sigma|=1$. Then $p_{0}=p=h([3 ; \mathrm{pp} .112-113])$ where $p$ is the order of the polynomial $P$, and so $\rho^{\prime}=1$. Consequently, the inequality (2.14) is satisfied for any $a^{\prime}>d$. Thus we have

THEOREM 2.2. For the petrowsky-parabolic system (2.8)-(2.9), suppose the initial function $u_{0}(x)$ belongs to the space $B_{\mu, d}$. Then for any small $\epsilon>0$, the correctness class of the system (2.8)-(2.9) belongs to the space $B_{\mu, d(1+\epsilon T)}$ for any $T>0$. 
Now let us consider for the case of negative genus. In this case according to $\left[2 ;\right.$ p. 210] and $\left[3 ;\right.$ p. 123], $\partial^{m} / \partial \sigma^{m} \exp \left(t p\left(-\sigma^{2}\right)\right)$ is majorized for $\operatorname{Re} \xi=\sigma$ by

$$
\left\|\frac{\partial^{k}}{\partial \sigma^{k}} \exp \left(t P\left(-\sigma^{2}\right)\right)\right\| \leqq C B^{k} k^{k\left(1-\sigma^{\prime} / 2 h\right)} \exp \left(-b^{\prime} t|\sigma|^{2 h}\right) .
$$

Since every testing function in the space $H_{\mu, \alpha}^{\beta}$ is of a form $x^{\mu+1 / 2} \varphi_{1}(x)$, where $\varphi_{1}$ is a smooth function on $0<x<\infty$, Lemma 1.2 and the inequality (2.15) show us that $\exp \left(t P\left(-\sigma^{2}\right)\right) \in x^{-(\mu+1 / 2)} H_{\mu, 1 / 2 h}^{\left(1-\rho^{\prime} / 2 h\right)}$. Let $\Phi$ belong to the space $\left.Y_{\mu, b}^{2 r}\right|_{z=x}$ (restriction to the real axis). Then

$$
\begin{aligned}
\sup _{-\infty<x<\infty} & \left|x^{2 k}\left(\exp \left(t P\left(-\sigma^{2}\right)\right)\right)\left(x^{-(\mu+1 / 2)} \Phi(x)\right)\right| \\
& \leqq \sup _{-\infty<\sigma<\infty}\left|\exp \left(t P\left(-\sigma^{2}\right)\right)\right| \sup _{-\infty<x<\infty}\left|x^{2 k}\left(x^{-(\mu+1 / 2)} \Phi(x)\right)\right|<\infty,
\end{aligned}
$$

where the last inequality is a consequence of (2.15) and Definition 1.3. It follows that $\exp \left(t P\left(-\sigma^{2}\right)\right)$ is a multiplier in the space $\left.Y_{\mu, b}^{2 r}\right|_{\xi=\sigma}$ for any $r>0, b>0$. Consequently, if the initial function $u_{0}(x)$ belongs to the space $B_{\mu, b}$ for any $b>0$, then $U(\sigma, t)=\exp \left(t P\left(-\sigma^{2}\right)\right) U_{0}(\sigma)$ belongs to the space $\left.Y_{\mu, b}^{2 r}\right|_{\xi=\sigma}$ and therefore $u(x, t)=\boldsymbol{h}_{\mu}^{-1} U(\sigma, t)$ belongs to the same space $B_{\mu, b}$ as $u_{0}(x)$ does. Continuous dependence of the solution on the initial function $u_{0}(x)$ is evident since $h_{\mu}^{-1} U(\sigma, t)$ is uniformly convergent with respect to $t$. Thus we have proved

THEOREM 2.3. Suppose the genus $\rho^{\prime}$ of the system $(2.8)^{\prime}-(2.9)^{\prime}$ is negative. If the initial function $u_{0}(x)$ belongs to the space $B_{\mu, b}$ for any $b>0$, then the correctness class of the system (2.8)-(2.9) belongs to the same space $B_{\mu, b}$ for $0 \leqq t \leqq T$, where $T=\left(2 h / b^{\prime} e\right) A^{1 / 2 h}$.

3. Hyperbolic systems. Consider a system of partial differential equations with constant coefficients

$$
\begin{gathered}
\frac{\partial u(x, t)}{\partial t}=P\left(S_{\mu}\right) u(x, t) \\
u_{0}(x)=u(x, 0)
\end{gathered}
$$

where $u(x, t)$ is an $m \times 1$ column vector, $S_{\mu}=D_{x}^{2}-\left(4 \mu^{2}-1\right) / 4 x^{2}$ as before, and $P(\xi)=\left(P_{j k}(\xi)\right)$ is an $m \times m$ matrix whose eigenvalues $\lambda_{j}(\xi)$ $(j=1,2, \cdots, s)$ are even powers of $\xi$. Let the system (3.1) be hyperbolic with $S_{\mu}$ replaced by $i \partial / \partial x$. Then for $\Lambda(\xi)=\max _{j} \operatorname{Re} \lambda_{l}(\xi)(\xi=\sigma+i \tau)$, 


$$
\begin{aligned}
& \Lambda(\xi) \leqq a|\xi|+b \\
& \Lambda(\sigma) \leqq C .
\end{aligned}
$$

The Hankel transform of the system (3.1)-(3.2) yields

$$
\begin{aligned}
\frac{\partial U(\xi, t)}{\partial t} & =P\left(-\xi^{2}\right) U(\xi, t) \\
U_{0}(\xi) & =U(\xi, 0) .
\end{aligned}
$$

Let $\lambda_{j}^{\prime}(\xi)\left(j=1,2, \cdots, s^{\prime}\right)$ be the eigenvalues of $P\left(-\xi^{2}\right)$ and let $\Lambda^{\prime}(\xi)=$ $\max _{1 \leqq j \leqq s^{\prime}} \operatorname{Re} \lambda_{j}^{\prime}(\xi)$. Then

$$
\begin{aligned}
& \Lambda^{\prime}(\xi) \leqq a^{\prime}|\xi|^{2}+b^{\prime} \\
& \Lambda^{\prime}(\sigma) \leqq C^{\prime} .
\end{aligned}
$$

Thus a proof similar to that of Theorems 2.1 or 2.2 leads us to

THEOREM 3.1. For $\mu \geqq-1 / 2$, let the initial data $u_{0}(x)$ belong to the space $B_{\mu, b}$ for any $b>0$. Then the correctness class of the hyperbolic system (3.1)-(3.2) belongs to the same space $B_{\mu, b}$ for $0 \leqq t \leqq T$ where $T$ is any positive real number.

4. Petrowsky-correct system. Consider a system of differential equations

$$
\begin{aligned}
\frac{\partial u(x, t)}{\partial t} & =P\left(S_{\mu}\right) u(x, t) \\
u_{0}(x) & =u(x, 0)
\end{aligned}
$$

where $u, P$ and $S_{\mu}$ are given as in $\S 3$. Suppose the system (4.1)-(4.2) is Petrowsky-correct with $S_{\mu}$ replaced by $i \partial / \partial x$. Let $\Lambda$ and $\Lambda^{\prime}$ be defined as before. Then

$$
\Lambda(\sigma) \leqq C, \quad \operatorname{Re} \xi=\sigma
$$

and

$$
\Lambda^{\prime}(\sigma) \leqq C^{\prime}, \quad \operatorname{Re} \xi=\sigma
$$

An argument similar to the proof of Theorem 2.1 yields

THEOREM 4.1. For any real numbers $\mu \geqq-1 / 2$ and $b>0$, let the initial data $u_{0}(x)$ belong to the space $B_{\mu, b}$. If the genus $\rho^{\prime}$ of the system 
$(3.1)^{\prime}-(3.2)^{\prime}$ is positive, then for all $0 \leqq t \leqq T, T>0$, the correctness class of the system (4.1)-(4.2) belongs to the space $B_{\mu, b+b^{\prime} T}$ where $b^{\prime}$ is arbitrarily near to $b$.

REMARK. Since $\quad\left\|\exp \left(t P\left(-\xi^{2}\right)\right)\right\| \leqq B\left(1+|\sigma|^{2}\right)^{h^{\prime}} \exp \left(b^{\prime} t|\tau|^{2 p_{d} \rho^{\prime}}\right)$ where $h^{\prime}$ is the correctness exponent of $(3.1)^{\prime}-(3.2)^{\prime}$ and $p_{0}$ is the reduced order of $P(\xi)$, we have to choose $r>p_{0} / \rho^{\prime}$ so that $U_{0}(\xi) \mapsto$ $\exp \left(t P\left(-\xi^{2}\right)\right) U_{0}(\xi)$ be a continuous linear mapping from $Y_{\mu, b}^{2 r}$ into $Y_{\mu, b+b^{\prime} T \text {. }}^{2 r}$

Suppose now the genus $\rho^{\prime}$ is nonpositive. Then for $\operatorname{Re} \xi=\sigma$,

$$
\left\|\frac{\partial^{k}}{\partial x^{k}} \exp \left(t P\left(-\sigma^{2}\right)\right)\right\| \leqq C_{k}\left(1+|\sigma|^{2}\right)_{k}^{r}
$$

where $r_{k} \leqq h^{\prime}+\rho^{\prime} k, h^{\prime}$ is given as before. Then the following theorem is a direct consequence of Theorem 2.3.

THEOREM 4.2. For any real numbers $\mu \geqq-1 / 2$ and $b>0$, let the initial data $u_{0}(x)$ belong to the space $B_{\mu, b}$. If the genus of the system $(3.1)^{\prime}-(3.2)^{\prime}$ is nonpositive, the correctness class of the system (4.1)-(4.2) belongs to the same space $B_{\mu, b}$ for all $0 \leqq t \leqq T$ where $T$ is any positive real number.

5. Concluding remarks. So far we showed that for given smooth initial data with compact support a correctness class of the differential operator $S_{\mu}$ with constant coefficients consists of smooth functions having compact support for any given $0 \leqq t \leqq T$. In the case of the differential operator $i \partial / \partial x$, Gelfand and Shilov ([3]) found a correctness class among nonsmooth functions via Fourier transforms and convolutions. Ineed their correctness class for small $t$ consists of ordinary functions of exponential type for parabolic and Petrowsky-correct system or $k$ times differentiable functions without any conditions at infinity for hyperbolic system. It is thus an open problem to find a correctness class of the differential operator $S_{\mu}$ among nonsmooth functions. The problem is equivalent to find conditions on the space of Hankel transformable functions with sutiable boundary conditions so that convolutions be allowable. As an example we were not able to identify the space of the convolution

$$
\boldsymbol{h}_{\mu}\left(x^{-(\mu+1 / 2)}\right) * \boldsymbol{h}_{\mu}\left(H_{\mu, 1 /(2 h)}^{1-\rho^{\prime}(2 h)}\right) \quad \text { for } \quad x>0
$$

in the proof of Theorem 2.2. 
Motivated by Schoenberg ([11]), Hirschman, Jr. ([6]) first introduced \#-convolution defined by

$$
(f \# g)(t)=\int_{0}^{\infty} \int_{0}^{\infty} f(x) g(y) D(t, x, y) d \nu(x) d \nu(y)
$$

where $f$ and $g$ are $L^{1}(0, \infty)$-functions with respect to the Radon measure $d \nu(x)=x^{2 \gamma}\left[2^{\gamma-1 / 2} \Gamma(\gamma+1 / 2)\right]^{-1} d x, y>0$,

$$
D(t, x, y)=2^{3 \gamma-5 / 2}[\Gamma(\gamma+1 / 2)]^{2}(t x y)^{-2 \gamma+1}\left[\Gamma(\gamma) \pi^{1 / 2}\right]^{-1}[\Delta(t, x, y)]^{2 \gamma-2}
$$

and $\Delta(t, x, y)$ is the area of the triangle whose sides are $t, x$ and $y$ if such a triangle exists otherwise is zero. With his definition of Hankel transform $\wedge$ given by

$$
f^{\wedge}(t)=\int_{0}^{\infty} f(x) g(x t) d \nu(x)
$$

where

$$
g(x)=2^{\gamma-1 / 2} \Gamma(\gamma+1 / 2) x^{1 / 2-\gamma} J_{\gamma-1 / 2}(x)
$$

he then showed that the \#-convolution is a counterpart of the *convolution for the Fourier transform. Further he proved that if $f$, $f^{\wedge} \in L^{1}(0, \infty), f$ and $f^{\wedge}$ are inverse to each other under the Hankel transform (5.1). Later on Haimo ([5]) showed that the space of \#convolutionable functions is an algebra with $L^{1}$-norm and thus the assumption that $f^{\wedge} \in L^{1}(0, \infty)$ is superfluous. Unfortunately their theories may not be applicable to our case since $x^{-(\mu+1 / 2)}$ does not belong to $L^{1}(0, \infty)$ with respect to $d \nu$. It is not known that every function in $\boldsymbol{h}_{\mu}\left(H_{\mu, 1 /(2 h)}^{1-\rho^{\prime}(2 h)}\right)$ is $L^{1}$-integrable with respect to $d \nu$.

\section{REFERENCES}

1. I. M. Gelfand and G. E. Shilov, Generalized Functions, vol. 1, Academic Press, 1967.

2. - Generalized Functions, vol. 2, Academic Press, 1968.

3. - Generalized Functions, vol. 3, Academic Press, 1967.

4. J. L. Griffith, Hankel transforms of functions zero outside a finite interval, J. Proc. Roy. Soc. New South Wales, 89 (1955), 109-115.

5. D. T. Haimo, Integral equations associated with Hankel convolutions, Trans. Amer. Math. Soc., 116 (1965), 330-375.

6. I. I. Hirschman, Jr., Variation diminishing Hankel transforms, J. Analyse Math., 8 (1960-61), 177-193.

7. L. Hörmander, Linear Partial Differential Operators, Springer-Verlag, 1969.

8. E. L. Koh and A. H. Zemanian, The complex Hankel and I-transformations of generalized functions, SIAM J. Appl. Math., 16 (1968), 945-957. 
9. W. Y. Lee, On spaces of type $H_{\mu}$ and their Hankel transformation, SIAM. J. Math. Anal., 15 (1974), 336-348.

10. - On the Cauchy problem of the differential operator $S_{\mu}$, Proc. Amer. Math. Soc., 51 (1975), 149-154.

11. I. J. Schoenberg, On totally positive function, Laplace integrals and entire functions of the Laguerre-Polya-Schur type, Proc. Nat. Acad. U.S.A., 33 (1947), 11-17.

12. E. C. Titchmarsh, The Theory of Functions, Oxford U. Press, 1964.

13. F. Treves, Topological Vector Spaces, Distributions and Kernels, Academic Press, 1967.

14. - Locally Convex Spaces and Linear Partial Differential Equations, Springer-Verlag, 1967.

15. Linear Partial Differential Equations with Constant Coefficients, Gordon and Breach, 1966.

16. G. N. Watson, A Treatise on the Theory of Bessel Function, Cambridge Univ. Press, 1966.

17. A. H. Zemanian, A distributional Hankel transformation, J. SIAM Appl. Math., 14 (1966), $561-576$.

18. The Hankel transformation of certain distributions of rapid growth, J. SIAM Appl. Math., 14 (1966), 678-690.

19. Hankel transforms of arbitrary order, Duke Math. J., 34 (1967), 761-770.

20. - Generalized Integral Transfromations, Interscience, 1968.

21. Distribution Theory and Transform Analysis, McGraw-Hill, 1965.

Received September 14, 1975.

Rutgers University - Camden Campus 


\section{PACIFIC JOURNAL OF MATHEMATICS}

\section{EDITORS}

RICHARD ARENS (Managing Editor)

University of California

Los Angeles, California 90024

\section{J. Dugundu}

Department of Mathematics University of Southern California Los Angeles, California 90007

D. Gilbarg and J. Milgram Stanford University Stanford, California 94305

\section{ASSOCIATE EDITORS}
E. F. BECKENBACH
B. H. NeumanN
F. WOLF
K. YoSHIDA

\section{SUPPORTING INSTITUTIONS}

UNIVERSITY OF BRITISH COLUMBIA CALIFORNIA INSTITUTE OF TECHNOLOGY

UNIVERSITY OF CALIFORNIA

MONTANA STATE UNIVERSITY

UNIVERSITY OF NEVADA

NEW MEXICO STATE UNIVERSITY

OREGON STATE UNIVERSITY

UNIVERSITY OF OREGON

OSAKA UNIVERSITY

\author{
UNIVERSITY OF SOUTHERN CALIFORNIA \\ STANFORD UNIVERSITY \\ UNIVERSITY OF HAWAII \\ UNIVERSITY OF TOKYO \\ UNIVERSITY OF UTAH \\ WASHINGTON STATE UNIVERSITY \\ UNIVERSITY OF WASHINGTON \\ AMERICAN MATHEMATICAL SOCIETY
}

The Supporting Institutions listed above contribute to the cost of publication of this Journal, but they are not owners or publishers and have no responsibility for its contents or policies.

Mathematical papers intended for publication in the Pacific Journal of Mathematics should be in typed form or offset-reproduced (not dittoed), double spaced with large margins. Underline Greek letters in red, German in green, and script in blue. The first paragraph or two must be capable of being used separately as a synopsis of the entire paper. Items of the bibliography should not be cited there unless absolutely necessary, in which case they must be identified by author and Journal, rather than by item number. Manuscripts, in duplicate, may be sent to any one of the four editors. Please classify according to the scheme of Math. Reviews, Index to Vol. 39. All other communications should be addressed to the managing editor, or Elaine Barth, University of California, Los Angeles, California, 90024.

100 reprints are provided free for each article, only if page charges have been substantially paid. Additional copies may be obtained at cost in multiples of 50 .

The Pacific Journal of Mathematics is issued monthly as of January 1966. Regular subscription rate: $\$ 72.00$ a year (6 Vols., 12 issues). Special rate: $\$ 36.00$ a year to individual members of supporting institutions.

Subscriptions, orders for back numbers, and changes of address should be sent to Pacific Journal of Mathematics, 103 Highland Boulevard, Berkeley, California, 94708.

PUBLISHED BY PACIFIC JOURNAL OF MATHEMATICS, A NON-PROFIT CORPORATION

Printed at Jerusalem Academic Press, POB 2390, Jerusalem, Israel.

$$
\begin{gathered}
\text { Copyright } 1976 \text { Pacific Journal of Mathematics } \\
\text { All Rights Reserved }
\end{gathered}
$$




\section{Pacific Journal of Mathematics}

Vol. 62, No. 2

February, 1976

Allan Russell Adler and Catarina Isabel Kiefe, Pseudofinite fields, procyclic

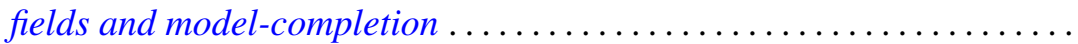

Christopher Allday, The stratification of compact connected Lie group

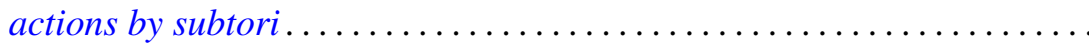

Martin Bartelt, Commutants of multipliers and translation operators .......

Herbert Stanley Bear, Jr., Ordered Gleason parts ..................

James Robert Boone, On irreducible spaces. II .....................

James Robert Boone, On the cardinality relationships between discrete

collections and open covers ............................

L. S. Dube, On finite Hankel transformation of generalized functions .......

Michael Freedman, Uniqueness theorems for taut submanifolds . . . . . . . . .

Shmuel Friedland and Raphael Loewy, Subspaces of symmetric matrices

containing matrices with a multiple first eigenvalue .............

Theodore William Gamelin, Uniform algebras spanned by Hartogs

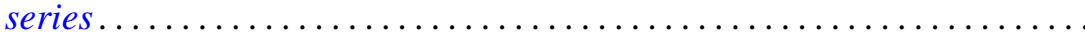

James Guyker, On partial isometries with no isometric part ............

Shigeru Hasegawa and Ryōtarō Satō, A general ratio ergodic theorem for

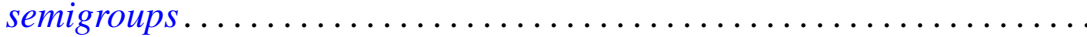

Nigel Kalton and G. V. Wood, Homomorphisms of group algebras with norm less than $\sqrt{2}$.

Thomas Laffey, On the structure of algebraic algebras...

Will Y. K. Lee, On a correctness class of the Bessel type differential operator $S_{\mu}$

Robert D. Little, Complex vector fields and divisible Chern classes ....

Kenneth Louden, Maximal quotient rings of ring extensions . .

Dieter Lutz, Scalar spectral operators, ordered $l^{\rho}$-direct sums, and the

counterexample of Kakutani-McCarthy . .

Ralph Tyrrell Rockafellar and Roger Jean-Baptiste Robert Wets, Stochastic

convex programming: singular multipliers and extended duality

singular multipliers and duality.

Edward Barry Saff and Richard Steven Varga, Geometric overconvergence of rational functions in unbounded domains ..........

Joel Linn Schiff, Isomorphisms between harmonic and P-harmonic Hardy

spaces on Riemann surfaces.

Virinda Mohan Sehgal and S. P. Singh, On a fixed point theorem of

Krasnoselskii for locally convex spaces.

Lewis Shilane, Filtered spaces admitting spectral sequence operations

Michel Smith, Generating large indecomposable continua . 